\title{
Reports of ICCM from 1998 to 2010
}

\section{International Congress of Chinese Mathematicians 1998 (Beijing)}

reported by Lo Yang

(Morningside Center of Mathematics, Chinese Academy of Sciences)

The first International Congress of Chinese Mathematicians was held from Dec. 12 to Dec. 16, 1998 at the Morningside Center of Mathematics, Chinese Academy of Sciences in Beijing. The conference was initiated by Prof.

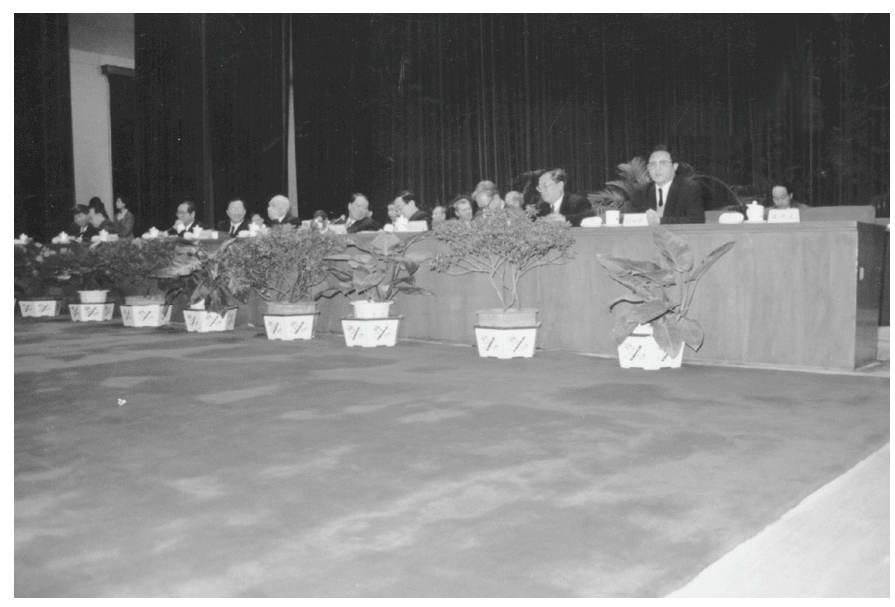

ICCM 1998 at Beijing

S.T. Yau of Harvard University, Fields medalist, member of the US National Academy of Sciences and foreign member of the Chinese Academy of Sciences. More than four hundred mathematicians from overseas, Hong Kong, Macau, Taiwan and mainland China participated in the conference. Among them were many excellent young mathematicians from China who went abroad to study in the 1980s, many well-established overseas Chinese mathematicians, more than thirty mathematicians from Taiwan, and more than forty mathematicians from Hong Kong and Macau, and more than two hundred mathematicians from the Mainland.

Several distinguished guests attended the conference: R. Graham (former President of the American Mathematical Society), J.P. Bourguignon (President of European Mathematical Society \& Director of Institut des Hautes
Etudes), J. Jost (Director of Max-Planck Mathematics Institute), M. Taylor (President of the London Mathematical Society), Seng Luan LEE (President of Mathematical Society of Singapore), T. Sunada (Treasurer of the Japan Mathe-

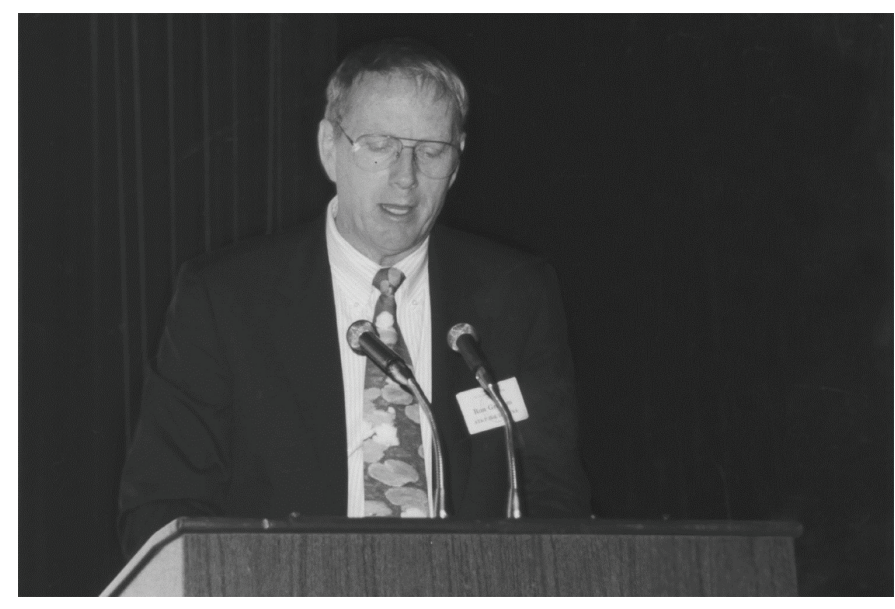

R. Graham

matical Society), and Kun Soo CHANG (President of Korean Mathematical Society).

The conference also invited the distinguished

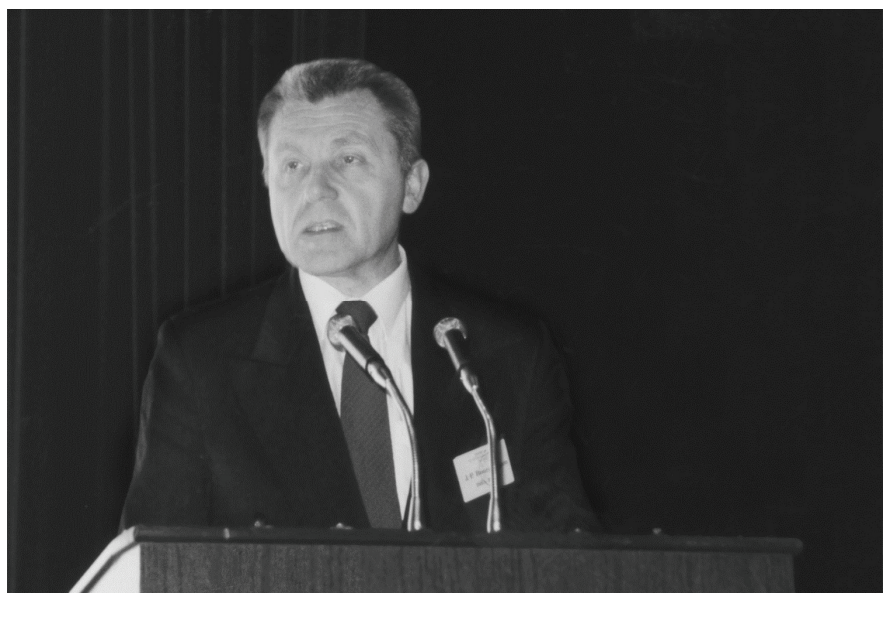

J.P. Bourguignon

mathematicians to deliver Morningside mathematics lectures. The lecturers were R. Borcherds (Cambridge University), J. Coates (Cambridge University) and R. Graham (Lucent Technologies). 
In the morning of the first day of conference, Dec. 12, 1998, the first Morningside medals award ceremony was held in the People's Great Hall. The following distinguished guests were invited to the ceremony: CHEN Siwei, Vice Chairman of the Standing Committee of the National People's Congress, ZHU Guang-Ya, Vice Chairman of the Chinese People's Political Consultative Conference, LU Yong-Xiang, President of the Chinese Academy of Sciences., Ronnie CHAN, Chairman of the Board of Trustees of the Morningside Group, ZHANG Yu-tai, the first General Secretary of Chinese Association of Science and Technology, WANG Nai-Yan, Deputy Director of the Na-

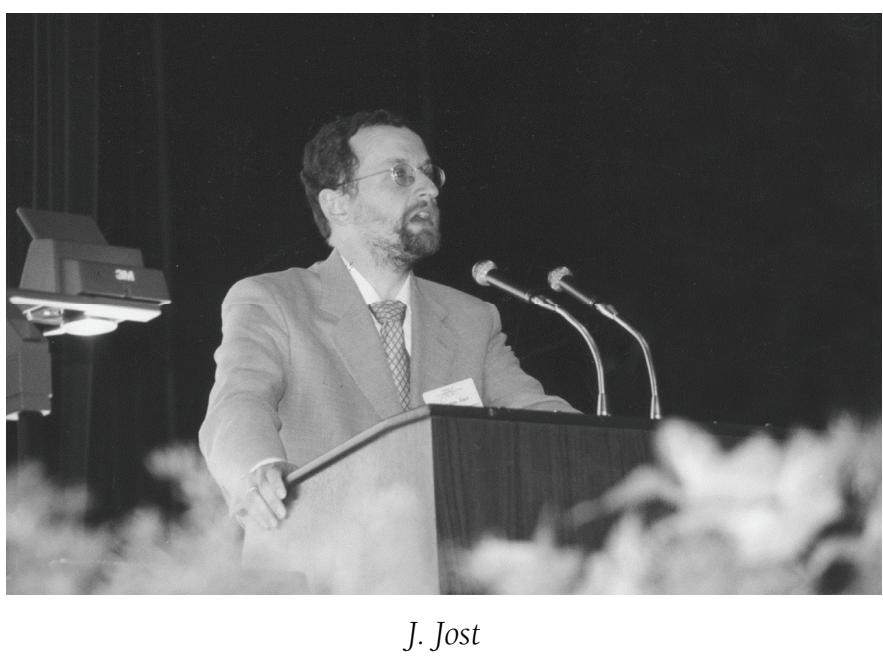

tional Science Foundation of China and DUAN Rui-Chun, Deputy General Secretary of the Ministry of Science and Technology of China.

The Morningside medals were established, through the Morningside Center of Mathematics, to encourage outstanding young mathematicians of Chinese descent in their pursuit of mathematical truth. The Morningside medals committee 1998 had the members: L. Carleson, J. Coates, R. Graham, M. Kashiwara, P. Lax and S.T. Yau (Chairman).

At the ceremony, it was announced that the two Gold Medal recipients were Chang-Shou LIN and Shouwu ZHANG, four Silver Medal recipients were Raymond CHAN, Chong-Qing CHENG, Kefeng LIU and Tong YANG. Chang-Shou LIN (Ph. D. of Courant Institute, NYU, 1984, Professor of Chung Cheng University and Director of the Center for Theoretical Sciences at Taiwan) was awarded the 1998 Morningside Gold Medal for his research work on locating isometric embedding problems, scalar curvature equations and semi-linear elliptic equations. Shouwu ZHANG (Ph. D. of Columbia University 1991, Professor of Columbia University) was awarded the 1998 Morningside Gold Medal for his contributions to the number theory and arithmetical algebraic geometry. His achievements include the connections between L-functions and arithmetic via the Gross-Zagier formula, the Galois distribution of points on Abelian varieties and pure Arakelov geometry. Raymond CHAN (Ph.D. of Cou- rant Institute NYU, 1985, Professor of Chinese University of Hong Kong) was awarded the 1998 Morningside Silver Medal for his work in computational mathematics. Chong-Qing CHENG (Ph.D. of Northwest Industrial University, 1987, Professor of Nanjing University) was awarded the 1998 Morningside Silver Medal for his contributions in the areas of dynamic systems and Hamiltonian dynamics. He is recognized for his work on problems related to Kolmogorov-Arnold-Moser (KAM) theory. Kefeng LIU (Ph.D. of Harvard University, 1993, Associate Professor of UCLA) was awarded the 1998 Morningside Silver Medal for his achievements in topology, geometry and mathematical physics. Tong YANG (Ph.D. of UC Davis, 1993 , Professor of City University of Hong Kong) was awarded the 1998 Morningside Silver Medal for his contributions to the fundamental well-posedness problem for general systems of hyperbolic conservation laws.

In the afternoon of December 12, the four Morningside Lectures were delivered in the conference hall of the Chinese Association of Science and Technology. Before the Morningside lectures, Mr. Ronnie Chan of the Morningside group, Hong Kong, delivered a speech on scientific development and was well received by the audience. He expressed his wish to give full support to the advancement of mathematics within China.

In addition, in the early morning of December 12, the newly finished Morningside Mathematics Building was dedicated by Prof. LU Yong-Xiang, the President of the Chinese Academy of Sciences, Mr. Ronnie CHAN, Chairman of the Board of Trustees of the Morningside Group

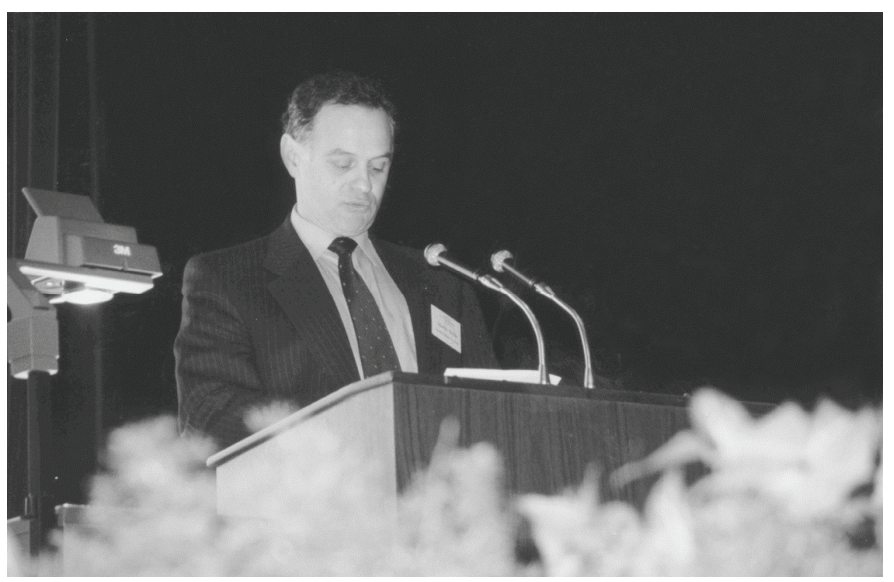

M. Taylor

and Prof. S. T. YAU of Harvard University. The Morningside Mathematics Building was funded partly by the Chinese Academy of Sciences.

In the evening of December 12, a concert was held in the hall of the Chinese Association of Science and Technology.

From December 13 to December 16, 1998, there were 13 one-hour plenary lectures and 55 invited 45-minute lectures, as well as more than 200 contributed talks in 13 sections: algebra and representation theory, algebraic 
geometry, number theory and automorphic forms, Riemannian geometry and geometric analysis, mathematical physics, topology, complex analysis and complex geometry, dynamical systems and control theory, elliptic and parabolic partial differential equations, hyperbolic parabolic partial differential equations, applied analysis, computational mathematics, and combinatorics.

The Proceeding of ICCM 98 was published by American Mathematical Society and International Press in 2001.

\section{The International Congress of Chinese Mathematicians 2001 (Taipei)}

\section{reported by}

Chang-Shou Lin

(National Taiwan University)

Song-Sun Lin

(National Chiao-Tung University)

The International Congress of Chinese Mathematicians (ICCM) was initiated by Prof. Shing-Tung Yau. The first ICCM conference was held in Beijing in 1998 and the second was held from Dec. 17 to Dec. 22, 2001 at the Grand Hotel, Taipei. The second conference was dedicated to Prof. Shiing-Shen Chern for his 90th birthday. Unfortunately, Professor Shiing-Shen Chern was unable to attend the conference due to health problems.

Several distinguished international mathematicians attended the second conference, such as John Coates (Cambridge University), Gerd Faltings (Max Planck Institute for Mathematics), Peter Lax (New York University), Masatake Mori (Kyoto University), Joel Smoller (University of Michigan, Ann Arbor), and Neil Trudinger (Australian National University). Among those distinguished attendees, John Coates, Masatake Mori, Joel Smoller and Neil Trudinger delivered Morningside Lectures. Gerd Faltings gave a plenary speech and Peter Lax gave a speech on "The Development of Applied Mathematics in the 21st Century."

In the morning of the first day of the conference, Dec. 17,2001 , after the opening address and welcoming remarks, the Morningside Lifetime Achievement Award in Mathematics was presented to professor Shiing-Shen Chern. The award was accepted by Professor Chern's daughter, May Chu (Pu Chern). Following the award, President Chung-Laung Liu of National Tsing Hua University presented S.S. Chern with an Honorary Doctorate Degree, which was also received by his daughter May Chu. Finally, Paul C.W. Chu, the son-in-law of S.S. Chern, read an address prepared by Professor S.S. Chern.

Professor S.S. Chern's former student Shing-Tung Yau gave the professor the following tribute:

Born in Jiaxing Zhejiang, Professor Shiing-Shen Chern is a world-class, mathematician who is highly respected by all distinguished scholars. He attained great academic success at an early age and rose rapidly to fame in Beijing and Shanghai. He spent his 30s and 40s studying abroad and doing advanced research, which earned him a strong reputation in Europe and the United States. Professor Chern focused on differential geometry and constructed the Chern classes. He also enhanced and developed the work of Cartan, and was a pioneer in topology.

Professor Chern has taught us how to learn from the old and to seek the new. He has nurtured so many scholars who have bestowed upon him their highest respect and praise. Even those Chinese scholars who study mathematics, but are not his student, have a great appreciation of Professor Chern.

We wish to thank him for his invaluable contributions of the past seventy years.

During the conference Gold Medal were awarded to Jun Li of Stanford University and Horng-Tzer Yau, New York University.

There were also four Silver Medals awarded that year. The silver medal recipients were: Daqing Wan (University of California, Irvine), Chin-Lung Wang, (National

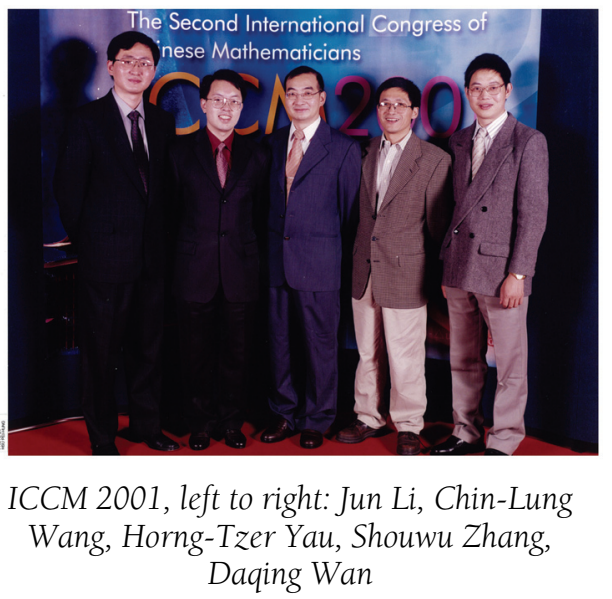

Tsing-Hua University, Taiwan), Sijue Wu (University of Maryland, College Park), and Nanhua Xi (Chinese Academy of Science, China).

The newly established S.S. Chern Prize was presented to Song-Sun Lin of National Chiao-Tung University for his contributions to mathematics and the mathematical community, and Jiu-Kang Yu of University of Maryland, College Park for his excellent work.

There were two activities in the afternoon: The first one was the symposium entitled "How Science and Technology Impact Asian Economies and Business," chaired by Ronnie C. Chan (Co-Founder, The Morningside Group and chairman, Hang Lung Group, Hong Kong) and Morris Chang (Chairman, Taiwan Semiconductor Manufacturing Co., Ltd., Taiwan) with panelists C. D. Tam (Executive Vice President, Motorola, inc. and President, Asia Pacific Region, Motorola Asia Pacific Ltd., Hong Kong) and Shih-Chien Yang (Chairman \& CEO, Global Strategic Investment Fund, Taiwan). 
The second activity was a conference entitled "The Development of Applied Mathematics in the 21st Century," chaired by Tai-Ping Liu (Director, Institute of Mathematics, Academia Sinica, Taiwan). The speakers for this activity included Peter D. Lax, Joel A. Smoller, Andrew Yao, Wing-Hung Wong, Thomas Yizhao Hou, and Weinan E.

From December 18 to 22, there were 6 Morningside Lectures, 18 plenary talks, 73 invited talks and 62 contributed talks were delivered in the 17 areas. There were usually around 300 to 500 participants attended for each talk.

\section{The International Congress of Chinese Mathematicians 2004 (Hong Kong)}

reported by

Ka-Sing Lau

(The Chinese University of Hong Kong)

In December 17-22, 2004, the Third International Congress of Chinese Mathematicians was held at The Chinese University of Hong Kong (CUHK). It was an honor for all of us as it was a recognition of the growth of strength of mathematical research in Hong Kong. It was also a challenge for us as it was the first time in Hong Kong to host such a large scale and high profile mathematics conference.

The planning was not without difficulty; one of the

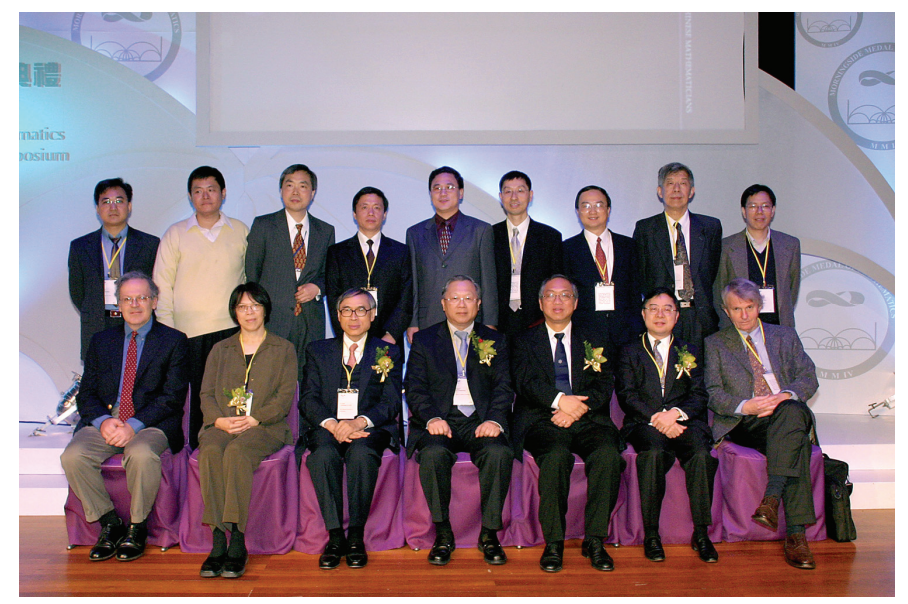

ICCM 2004 at Hong Kong

problems we faced was that there was no public research funding available for such activity in Hong Kong. We were grateful that all the sister mathematics departments and colleagues were very supportive and contributed to the event. Professor Shing-Tung Yau and the local organizing committee also spent over a year to solicit the possible donors and private funding agencies. We appreciated very much that Dr. Ronnie Chan and the Morningside Group took up a large part of expenses, and put in a lot of man-power to help the detail arrangement in the opening ceremony.

The opening ceremony was held in the Exhibition Hall of Hong Kong, it was attended by a thousand participants including fellow mathematicians, friends and supporter of the ICCM. Professor Shing-Tung Yau presided over the ceremony, he proclaimed to dedicate the Congress to the memory of the great mathematician and teacher, Professor Shiing-Shen Chern, who passed away just before the meeting. Professor Yongxiang Lu, the President of the Chinese Academy of Sciences, was the Honorable President of the Congress, and he presented the Morningside Gold Medals and Silver Medals to the group of distinguished mathematicians, Professors Kefeng Liu, Zhouping Xin , Thomas Yizhao Hou, Zhiliang Ying, Jin-Yi Cai, Aiko Liu, Xi-Ping Zhu.

For their accomplishments, Kefeng Liu (Ph.D. of Harvard, Professor of UCLA) was awarded the Morningside Gold Medal of Mathematics for his work on Witten's rigidity theorem and elliptic genus. Zhouping Xin (Ph.D. of Michigan, M.W. Mong Professor of Mathematics at CUHK) was awarded the Morningside Gold Medal of Mathematics for his work on nonlinear partial differential equation and boundary layer theory, Prandtl equations and shock wave flows. Thomas Yizhao Hou (Ph.D. of UCLA, Charles Lee Powell Professor of Applied and Computational Mathematics at Caltech) was awarded the Morningside Gold Medal of Applied Mathematics for his work on the point vortex method, numerical methods for fluid interfaces with surface tension, analysis of three dimensional vortex sheets, and the singularity criteria for the three dimensional Euler equation. Zhiliang Ying (Ph.D. of Columbia, Professor of Statistics at Columbia) was awarded the Morningside Gold Medal of Applied Mathematics for his work on survival analysis, counting processes, adaptive designs, and applications to AIDs research, psychological measurement and econometrics. Jin-Yi Cai (Ph.D. of Cornell, Professor of Computer Science at Wisconsin) was awarded the Morningside Silver Medal of Mathematics for his work on the resolution of two long-standing open conjectures on sparse sets, and other important discoveries in computational complexity. Ai-Ko Liu (Ph.D. of Harvard, Assistant Professor at UC Berkeley) was awarded the Silver Medal for his work on Seiberg-Witten theory and the topology of symplectic 4-manifolds. Xi-Ping Zhu (Ph.D. of Chinese Academy of Sciences, Professor at Zhongshan University) was awarded the Morningside Silver Medal of Mathematics for his work on Ricci flow on Kähler manifolds, and the complete Kähler manifolds of positive curvature.

The Chern Prize was awarded to Professors Lo Yang and Fang-Hua Lin. The award to Professor Yang (Member of the Chinese Academy of Science, founding director of the Academy of Mathematics and System Sciences) was for his dedication to the development of mathematics in China and for his role in promoting mathematical activities endeavored over the past twenty years. His had far 
reaching contributions in complex analysis included the deficient values and Borel directions of entire and meromorphic functions, and the solution to a conjecture of Littlewood. The award to Professor Lin (Ph.D. of Minnesota, Silver Professor of Mathematics at Courant Institute, NYU, member of American Academy of Arts and Sciences) was for his fundamental contributions to the theory of liquid crystals, harmonic maps Ginzburg-Landau equations, the static as well as dynamic theory of topological

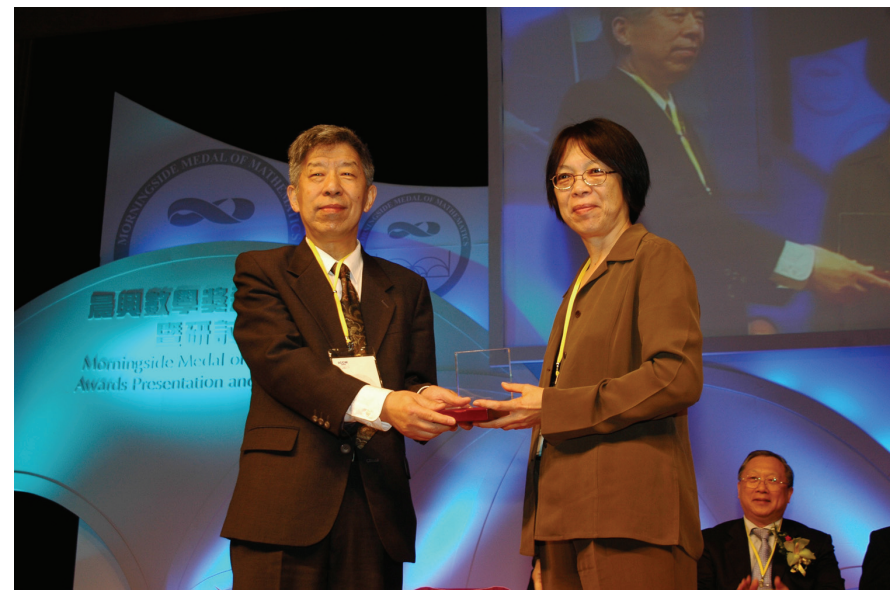

Lo Yang and May Chu, the daughter of Shiing-Shen Chern

defects, Skyrme and Faddeev models, and the Navier-Stokes equations.

Also in recognition of the overseas friends who had made great contributions to the mathematics communities in China, the Congress instituted a new award called the ICCM International Cooperation Medal for Mathematics, and the laureate was Professor John Coates. Professor Coates (Sadleirian Professor of Pure Mathematics at Cambridge) generously gave his knowledge and time to Chinese mathematics in the past twenty years; he trained many Chinese students and post-doctoral fellows, and had been a tremendous influence on their growth as mathematicians.

The academic program was held in CUHK campus, and was attended by seven hundred mathematicians. It covered many areas including pure mathematics, applied mathematics, statistics, and computer sciences. The Chern Lecture was given by Professor Shing-Tung Yau. There were five Morningside Lectures by Professors John Coates, Stanley Osher, Wilfried Schmid, Joel Smoller, and Leslie Valiant. In addition, there were twenty plenary lectures, two hundred fifty invited talks and contributed talks.

The purpose of the ICCM is to offer a platform to encourage the exchange of ideas between mathematicians of Chinese descend all over the world, and to honor those with the most outstanding accomplishments. The ICCM in Hong Kong has definitely achieved this goal with great success.

\section{International Congress of Chinese Mathematicians 2007 (Hangzhou)}

reported by Hong-Wei Xu

(Center of Mathematical Sciences, Zhejiang University)

The fourth International Congress of Chinese Mathematicians (ICCM) was held from Dec. 17 to Dec. 22, 2007 at Zhejiang Great Hall of the People and Zhejiang University in Hangzhou, with about fifteen-hundred participating mathematicians. Although most of them were Chinese or Chinese descent, many participants arrived as well from America, Europe, India, Japan, and Korea. This congress was sponsored by the Morningside Group founded by the brothers Ronnie and Gerald Chan, Zhejiang University, and Professor Shing-Tung Yau. The congress president was Professor Shing-Tung Yau, Fields medalist, member of the US National Academy of Sciences and foreign member of the Chinese Academy of Sciences. The organizing committee of the congress was chaired by Professor Kefeng Liu of Zhejiang University and UCLA.

On the opening day of the congress, Dec. 17, 2007, the Morningside Gold Award for Mathematics was presented to Professor Xu-Jia Wang of Australian National University, and the Morningside Gold Award for Applied Mathematics went to Professor Jianqing Fan of Princeton University. The five Morningside Silver Awards were given to Professor Chiun-Chuan Chen of Taiwan University, Professor Lizhen Ji of University of Michigan, Professor Shi Jin of University of Wisconsin-Madison, Professor

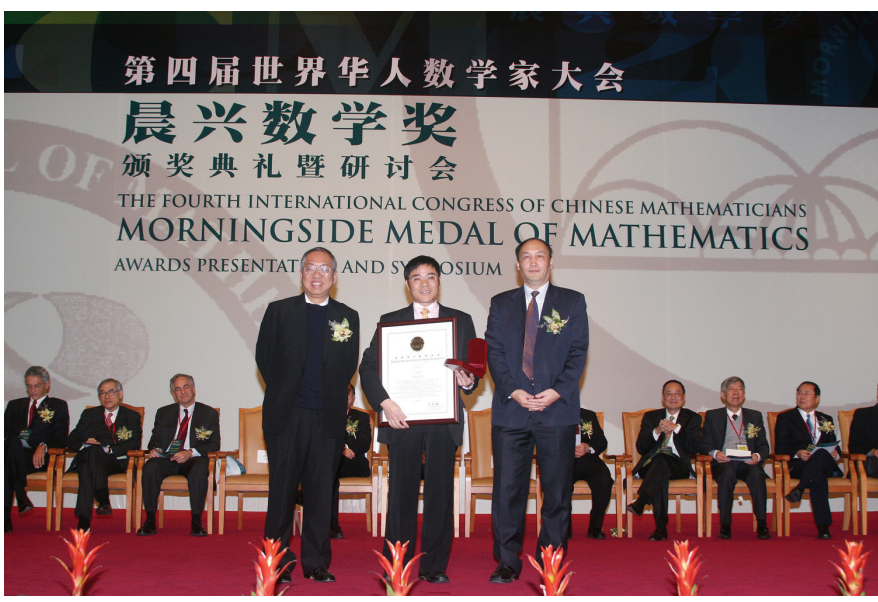

Jianqing Fan was awarded the 2007 Morningside Gold Medal of Applied Mathematics

Melissa Chiu-Chu Liu of Northwestern University and Columbia University, and Professor Ye Tian of Morningside Center of Mathematics, CAS. The Chern Awards were presented to Professor Shiu-Yuen Cheng of Hong Kong University of Science and Technology, and to Professor Mu-Tao Wang of Columbia University. The International Cooperation Award went to Professor Stanley J. Osher of University of California, Los Angeles. The governor of Zhejiang province Zushan Lv and other officials from the 
city and the province came to the congress. We also presented letters from government officials who had written to express their support for the congress, including minister Yuan-Chao Li, Yongxiang Lu (vice chairman of the National People's Congress, president of the CAS), and Hongzhu Zhao (secretary of the party of Zhejiang Prov-

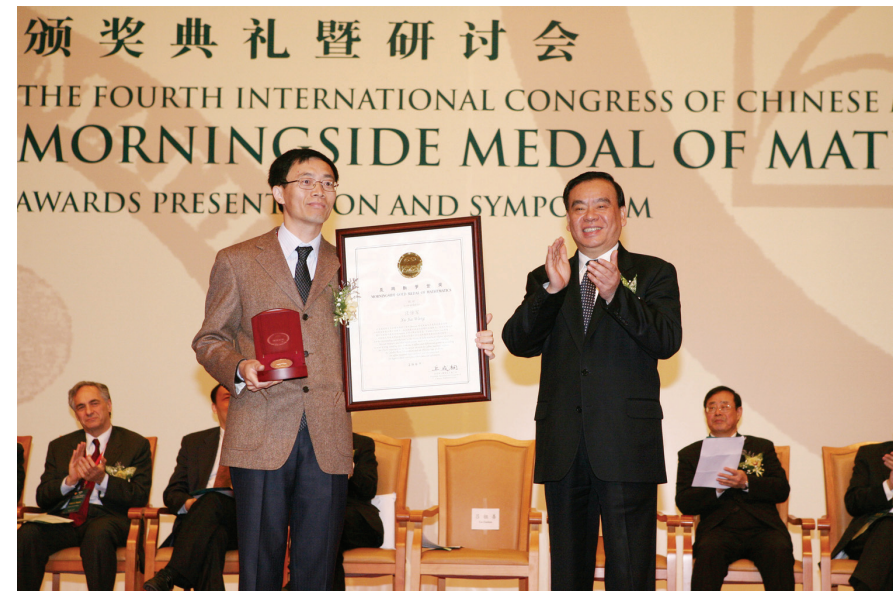

Xu-Jia Wang (the left) with the 2007 Morningside Gold Medal of Mathematics

ince).

A new award was also presented at ICCM 2007. The New World Mathematics Awards, sponsored by the New World Development Company, recognizes outstanding doctoral, masters and undergraduate theses written by mathematicians of Chinese descent who have graduated from universities and institutes in the past three years. The Gold Awards for Doctor Thesis in 2007 went to Dr. Hao Xu of Zhejiang University (Advisor: Professor Kefeng Liu) and to Dr. Weizhe Zheng of University of Paris XI (Advisor: Professor Luc Illusie). Another new award was established at ICCM 2007. S.-T. Yau High School Mathematics Awards, sponsored by Taikang Life Insurance, recognizes excellence in mathematics research projects among high school students of Chinese descent throughout the world.

A symposium, held in the afternoon of the opening day, featured top mathematicians and university presidents who examined mathematics-related topics. The first session looked at the challenges facing higher education in the twenty-first century. Panelists include former Dean of Harvard College Benedict Gross; Chancellor of the Chinese University of Hong Kong Lawrence Lau; Dean of the Graduate School of Arts and Sciences at Columbia University Henry Pinkham; Chancellor of the University of California at Santa Barbara Henry Yang; President of Zhejiang University Wei Yang; and President of the University of Chicago Robert Zimmer.

The second session focused on how mathematics was playing an increasingly important role in biomedical science. Jill Mesirov, the chief informatics officer at the Broad Institute, provided an overview of the latest cut- ting-edge developments in genomics and biomedical research.

The final session examined the critical issues facing primary and secondary mathematics education in Europe and the United States. Professor Wilfried Schmid of Harvard University and Professor Jean-Pierre Demailly of Universite de Grenoble I shared their thoughts and the ensuing discussion was chaired by Professor Hung-Hsi $\mathrm{Wu}$ of the University of California at Berkeley. The discussion was carried over to the next day, when a new panel convened to examine mathematics education in Mainland China.

A panel discussion on women mathematician was held in the evening on Dec. 19, 2007. Panelists include Yunmei Chen (University of Florida), Fan Chung Graham (UC San Diego), Min Ji (Chinese Academy of Sciences), Yng-Ing Lee (Taiwan University), Wen-Ching Li (Pennsylvania State University), Melissa Chiu-Chu Liu (Columbia University), Jianghua Lu (Univ of Hong Kong), Dusa McDuff (Stony Brook University), Chuu-Lian Terng (UCI), Claire Voisin (CNRS France), Guofang Wei (UC Santa Barbara).

From December 18 to December 22, 2007, there were 17 one-hour plenary lectures, and 198 invited 45-minute talks in 40 sessions. Thirty five distinguished mathematicians delivered special addresses at the congress, including twelve Morningside lectures, nine international distinguished lectures, six New World lectures, seven Taikang lectures and one Mui's lecture. The Proceedings of ICCM 2007 were published by the Higher Education

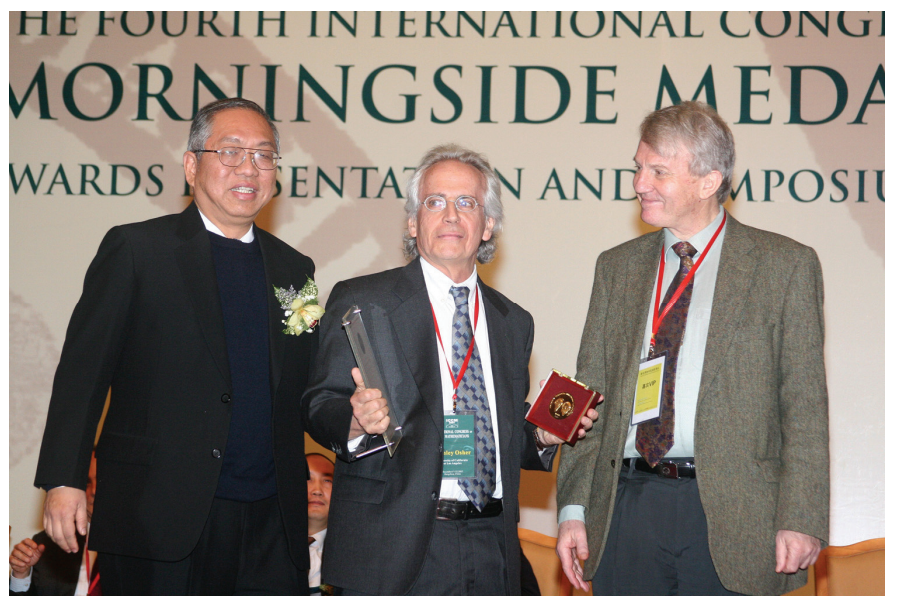

Left to right: Shing-Tung Yau, Stanley J. Osher and John Coates

Press and the International Press before the congress.

ICCM 2007 attracted more than thirty mainstream media to Hangzhou for interview reports. CCTV, China Education Television Station, Zhejiang TV Station, Shanghai TV Station, Xinhua News Agency, People's Daily, Guangming Daily, Wen Wei Po, Zhejiang Daily, Hangzhou Daily, Science Times and other mainstream media made the detailed reports and interviews for the congress. CCTV broadcast news about the Opening Ceremony for 
the Fourth ICCM on December 17, 2007, which brought out huge influence in China.

\section{International Congress of Chinese Mathematicians 2010 (Beijing)}

\section{reported by}

Yat Sun Poon

(University of California at Riverside)

As one-thousand-five-hundreds mathematicians from around the world gradually converged at the Great Hall of the People in Beijing in the chilly morning of December 17, 2010, the fifth International Congress of Chinese Mathematicians commenced. These mathematicians come from all over China and from around the world. While most of the participants are Chinese, there are a large number of mathematicians from all nationality. Most of the participants were professional mathematicians. They were also a substantial number of graduate students. They all came to celebrate mathematics and the success of their peers. They all came to admire the heroes of the past generation and to celebrate the success this generation. After the opening ceremony, they will all share their individual achievements and ideas in a week-long conference in Tsinghua University.

After twelve years, the ICCM returned to Beijing. As one of many organizers of this event, I was amazed to see that the growth of ICCM's scientific program is in direct proportion to the growth of China's economic strength during the same period of time. Mathematics is the entry point for modern technology, sciences, economics and finance. A nation's ability to compete at international level is in part reflected by the depth, breadth and quality of its talent pool in mathematics. To bring mathematics in China and Chinese mathematicians to their current stage of development, many people have made profound contributions in various situations and levels. Among all Chinese mathematicians of the past one hundred years, Loo-Keng Hua and Shiing-Shen Chern stood out as two giants. They both made great contributions to mathematics, mathematics education, and application of mathematics. Their impact has not only been scientific, but also institutional. Therefore, it is most fitting for ICCM2010 to be dedicated to Hua's centennial birthday and Chern's ninety-ninth birthday.

After the chair of ICCM, Prof Shing-Tung Yau, welcome the participants, the assembly bestowing the Morningside Gold Medal of Mathematics to Professor $\mathrm{Mu}$-Tao Wang of Columbia University and Professor Sijue $\mathrm{Wu}$ of University of Michigan at Ann Arbor. The assembly also bestowed the Morningside Gold Medal of Applied Mathematics to Jun S. Liu of Harvard. Just like all previous awardees, these three young mathematicians stood on the shoulder of giants and are respected by a committee of the most distinguished mathematicians.
Chaired by S-T Yau, the selection committee was consisted of the following members: John $\mathrm{H}$. Coates (Cambridge), Simon K. Donaldson (Imperial College), Gerd Faltings (Max Planck Institute for Mathematics), Jean-Marc Fontaine (University of Paris-Sud), Benedict H. Gross (Harvard), Nigel Hitchin (Oxford), Stanley J. Osher (University of California, Los Angeles), Richard M. Schoen, (Stanford), Joel A. Smoller (University of Michigan, Ann Arbor) and Grace Wahba (University of Wisconsin, Madison).

While mathematics is the focus of all achievement, the ICCM also recognized those who made exceptional effort to serve its community through the Chern prize. The recipients for this award in 2010 were Professor Jiaxing Hong of Fudan University, Wen-Ching Li of National Tsing Hua University and Conan Leung of the Chinese University of Hong Kong.

Professor Hong is well known for his systemic study of isometric embedding of surfaces into Euclidean

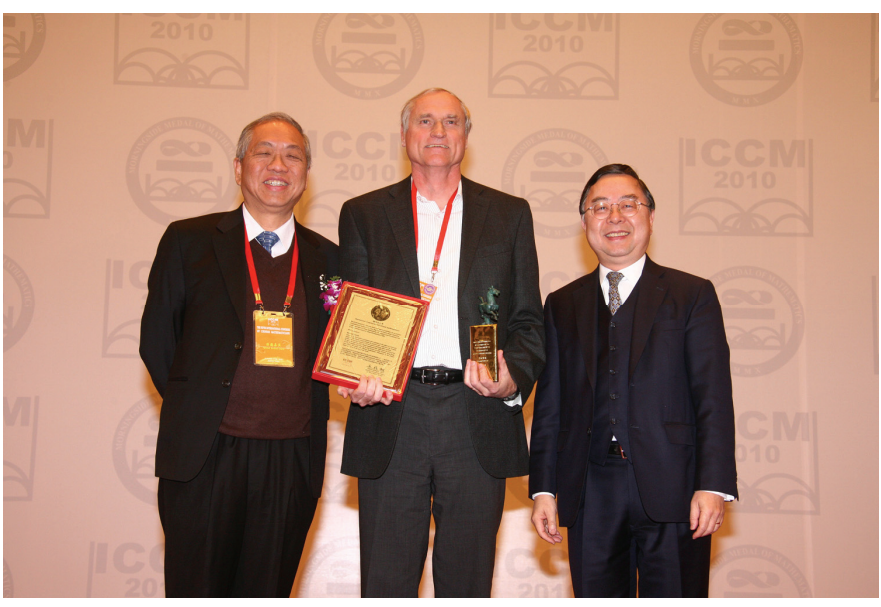

Left to right: Shing-Tung Yau, Richard Schoen (the awardee of the International Cooperation Award) and Ronnie C. Chan

three-dimensional space and his proof of a series of existence and regularity that have far reaching consequences, Professor Hong is also known to make every effort to help and nurture young mathematicians in China. Professor Wen-Ching Winnie Li is an internationally renowned number theorist. In the last two decades, with her expertise on number theory, she works beautifully into the frontiers of spectral graph theory and coding theory. She has inspired many young mathematicians in Asian Pacific region through her excellent courses and lectures during the past 25 years. Currently she serves as the director of the National Center for Theoretic Sciences in Taiwan. Together with her predecessors, they promote mathematical researches in Taiwan monumentally. On the other hand, Professor Conan Nai-Chung Leung is honored for significant research contributions in the study of mirror symmetry and quantum cohomology, and for his contribution to the community through his leadership in organizing many professional events. 
While the ICCM is conceived as a venue for interaction of Chinese mathematicians, it enjoys the supports and participants of mathematicians from around the world. It is evident from the composition of the selection committee of the Morningside Prize each year. Among all these mathematicians, some made particularly substantial contribution over a long period of time to the development of Chinese mathematics. They are honored by the International Cooperation Award. The awardee for 2010 is Professor Richard Schoen of Stanford University. Professor Schoen has cultivated closed relations with Chinese mathematicians throughout his career. More than one third of Professor Schoen's thirty-four Ph.D. students and many postdoctoral researchers are from mainland China, Hong Kong and Taiwan.

The opening ceremony is followed by a five-day intensive conference in Tsinghua University. This conference was fully supported by the administration, professors and students of Tsinghua University. The faculty, staff and students of its Mathematical Sciences Center and Department of Mathematical Sciences devoted lots of effort and time to make this event a success. In the morning, lectures were delivered by members of Morningside Medal Selection Committee, distinguished international mathematicians, statisticians and mathematical physicists from around the world. It is combined with over twenty one-hour talks by the Morningside Medalists and some of the most distinguished Chinese mathematicians in the morning. In five afternoons, nine workshops took place in parallel. Over one-hundred twenty speakers presented their latest discovery.

A feature of ICCM2010 was that it was enveloped by a sequence of mathematics events. While the Morningside Medals honor some of the best Chinese mathematicians at the age forty-five and below, talents should be articulated and nurtured at every stage of their research career. In 2010, Professor Shing-Tung Yau completed his construction of a vertical infra-structure to honor the research accomplishment by high school students, undergraduate students, master degree students and Ph. D degree students. It is complemented by a program to discover those who are most likely to succeed in graduate student mathematics. Immediately after the ICCM2010 was concluded, with the support of Tsinghua University and its Mathematical Sciences Center, some of the ICCM mathematicians joined another group of distinguished from around the world to inaugurate the Tsinghua-Sanya International Mathematics Forum in Hainan.

The S-T Yau High School Mathematics Awards (YHMA) was established in 2008 by S-T Yau with the financial support of Taikang Life Insurance Company Ltd.. The purpose of YHMA is to discover and nurture young mathematical talents. It encourages high school students in their creativity and originality. The organization and selection mechanism refers to "Siemens Westinghouse Science and Technology Competition." With its base in China, this program serves students worldwide. Each year, hundreds of entries were screened at the local level before they reach the final presentation. In 2010, the final presentation took place on Dec 15-16, immediately before the ICCM2010 in Tsinghua University. Teams from China, US, Singapore and Vietnam presented their projects in front of a distinguished panel of mathematicians. The panelists were Shing-Tung Yau (Harvard University), Neil Trudinger (Australian National University), John Coates (University of Cambridge), Jean-Marc Fontaine (Université Paris-Sud 11), Benedict Gross (Harvard university), Joel Smoller (University of Michigan), Eduard Looijenga (Universiteit Utrecht), Stanislaw Janeczko (Warsaw University of Technology), Ari Laptev (Imperial College London), Duong H. Phong (Columbia University), Hyam Rubinstein (University of Melbourne), Ching-Li Chai (University of Pennsylvania), Kefeng Liu (University of California, Los Angeles), Shiu-Yuen Cheng (Hong Kong University of Science and Technology) and Richard Schoen (Stanford University).

The gold award was given to Boyu Chen of Shanghai Shibei High School. The title of his research project was "A Proof on the Non-differentiability of Weierstrass Function in An Uncountable Dense Set."

In 2007, Dr. Henry Cheng, Managing Director of New World Development Company Limited and Professor Shing-Tung Yau, co-initiated the "New World Mathematics Awards" (NWMA). The goal of this program is to encourage outstanding Chinese mathematics students worldwide in research mathematics. This program honors the best thesis written by Chinese students worldwide. Awards are given at three different levels, namely, undergraduates, master degree, and Ph. D. degree. The NWMA ceremony takes places once every three years, in concurrent with ICCM. Award ceremony of 2010's NWMA was held at Beijing Hotel on December 17th, 2010. The members of international committee for 2010 were Shing-Tung Yau (Harvard University), Shiu-Yuen Cheng (Hong Kong University of Sciences and Technology), John Coates (University of Cambridge), Benedict Gross (Harvard University), Stanley Osher (University of California, Los Angeles) and Duong Phong (Columbia University).

The Gold Awards for Doctor Thesis in 2010 went to:

- $\quad$ Chen-Yu Chi, Harvard University

Thesis title: The pseudonorms and theorems of

Torelli type for birational equivalence

Advisor: Professor Shing-Tung Yau

- $\quad$ Yongquan Hu, Université Paris-Sud 11

Thesis title: On p-adic and modulo plocal Langlands program

Advisor: Professor Christophe Breuil

- Yu-Jong Tzeng, Stanford University

Thesis title: A proof of the Göttsche-Yau-Zaslow

formula

Advisor: Professor Jun Li 
- Jun Yin, Princeton University

Thesis title: Quantum many-body systems with

short-range interactions

Advisor: Professor Robert Seiringer

In 2010, S-T Yau completed his construction of a vertical infrastructure. He proposed to establish the S-T Yau College Student Mathematics Contests (YCMC). This program is open to all college students from mainland China, Hong Kong, and Taiwan. It purpose of this program is to elevate Chinese students' ability to performance in the early years of their graduate school careers. After a round of written tests at the level of the qualifying examination of top US graduate programs, a collection of high performing students is asked to participate in oral examinations. Result of YCMC has become important reference for admission to graduate programs worldwide. The first YCMC took place in Tsinghua University, Beijing on December 14, 2010. An award ceremony was held in the Royal King Hotel in the evening of December 20, 2010. The international panelists for a final decision were Shing-Tung Yau (Harvard University), Ben Andrews (Australia National University), John H. Coates (Cambridge University), Duong H. Phong (Columbia University), Benedict H. Gross (Harvard University), Jun Liu (Harvard University), Kefeng Liu (University of California, Los Angeles), Kenneth A. Ribet (University of California, Berkeley), Chi-Wang Shu (Brown University), Richard M. Schoen (Stanford University)

With the participation of all the professional research mathematicians, graduate students and high school teachers and students, it was no doubt that ICCM 2010 was a great success. The ICCM 2013 will soon take place in Taipei. One should of course expect that the ICCM will once again be a venue to celebrate mathematics and mathematicians. However, with the formation of the International Consortium of Chinese Mathematics, this triennial meeting now has evolved into an organization to promote mathematics and mathematics education at their highest level.

\section{Job Opportunity at the Mathematical Sciences Center, Beijing}

The Mathematical Sciences Center (MSC) of Tsinghua University is a leading center in China. It was inaugurated in December 2009. By fall 2013, it has over fifteen full-time members, six post-doctoral fellows, and over sixty fellows visited for at least one month. John Erik Fornaess of University of Michigan, Shiu-Yuen Cheng of The Hong Kong University of Science \& Technology and Eduard Looijenga of Utrecht will join MSC full-time in 2013. Spencer Bloch of University of Chicago will teach a course each year in the next ten years. In the coming years, MSC will continue to expand its faculty hiring and graduate student recruitment. For MSC activities, please visit http://msc.tsinghua.edu.cn/. 